Europhysics Letters

PREPRINT

\title{
Observation of narrow fluorescence from doubly-driven four-level atoms at room temperature
}

\author{
Uday Kumar Khan, Jimmy Sebastian, N. Kamaraju, Andal Narayanan, \\ R. SRINIVASAN AND Hema RAMAChandRan \\ Raman Research Institute, Sadashivnagar, Bangalore, INDIA 560080
}

\begin{abstract}
. -
Unusually narrow fluorescence peaks are seen from ${ }^{85} R b$ atoms under the action of two driving laser fields that are in a three-dimensional molasses configuration. One of the lasers is held at a fixed detuning from the "cooling" transition, while the other is scanned across the "repumping" transitions. The fluorescence peaks are split into symmetric pairs, with the separation within a pair increasing with the detuning of the cooling laser. For large detunings, additional small peaks are seen. A simple model is proposed to explain these experimental observations. An exhaustive density-matrix calculation of the four level system is presented in a companion paper.
\end{abstract}

Introduction. - Cooling of alkali atoms from room temperature to a few hundred microkelvin using near-resonant light has now become a standard technique for obtaining cold atoms. An area of current interest is the response of the cold atoms to multiple laser fields. The response may be incoherent, giving rise, for example, to sequential transitions, or coherent, resulting in quantum interference effects like Electromagnetically Induced Transparency (EIT) [2], Coherent Population Trapping, Lasing Without Inversion (LWI) [3] etc. Underlying most of these phenomena is the fundamental response of an atom to a strong pump beam, in the form of ac Stark splitting of the electronic levels. Also known as Autler-Townes dressed states [4], these manifest themselves as modifications to the absorption spectrum. A large volume of theoretical work on the absorption and emission from three level atoms under the action of pump and probe fields exists [5] and several experimental studies of the absorption spectra that verify some of the predictions have been reported [6]. However, there are very few [7] investigations of the fluorescence from four level systems under the action of several fields.

We report in this paper, fluorescence measurements from a doubly driven four level system. The alkali atom ${ }^{85} \mathrm{Rb}$ under the action of two laser fields, the "cooling" and "repumper" fields, in a three dimensional optical molasses configuration is studied. Unusual features are seen in the fluorescence when one of the lasers, the "cooling" laser is held at fixed frequency, and the other scanned. We discuss in simple physical terms, a mechanism that gives some insight into the experimental observations. Detailed theoretical analysis in terms of a four-level density matrix is presented in a companion paper [1].

(c) EDP Sciences 


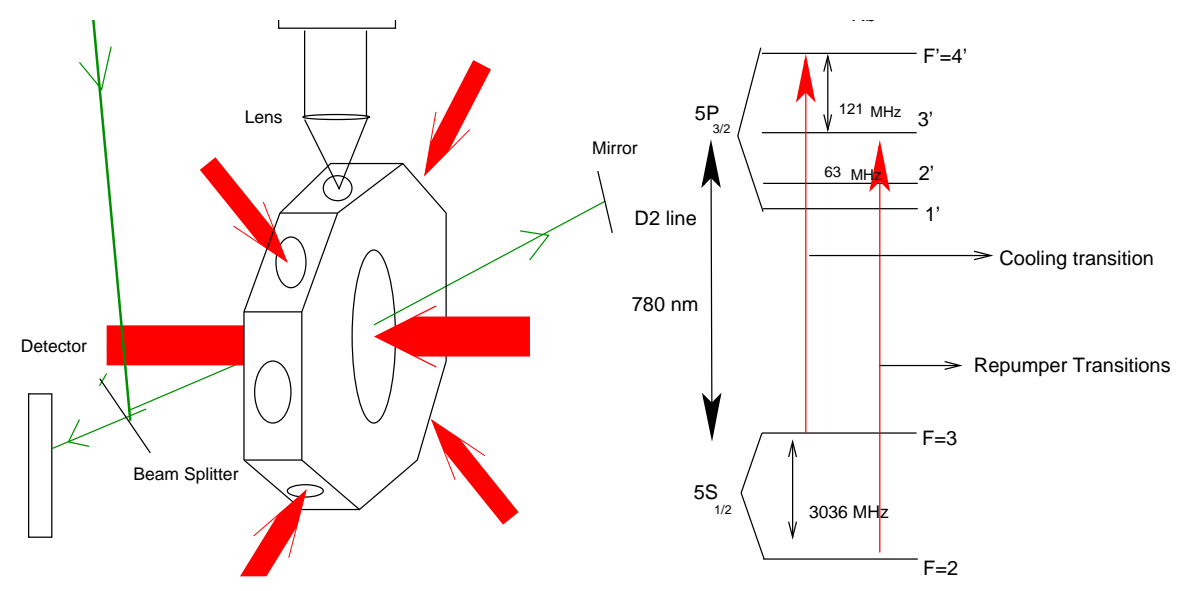

Fig. 1 - The experimental setup : the thick red lines are the cooling and repumper beams, the green line the absorption probe. The energy level diagram of ${ }^{85} R b$ is given on the right.

Experiment. - The energy levels of ${ }^{85} R b$ (Fig. 1) show that the closed transition $5 S_{1 / 2} F=3->5 P_{3 / 2} F^{\prime}=4\left(^{1}\right)$ is appropriate for cooling. As is well known, even when the cooling laser is resonant with the cooling transition, due to their proximity adjacent hyperfine levels also get populated. These levels can then decay, in addition to the $\mathrm{F}=3$ level, to the other ground level $\mathrm{F}=2$ also, thus depleting the cooling cycle in a few absorption-emission cycles. This necessitates the use of a repumper laser, tuned to the $\mathrm{F}=2->\mathrm{F}^{\prime}=3$ or 2 , transition which serves to bring back atoms to the $\mathrm{F}=3$ state, and thus into the cooling cycle.

In our experiment we use two external cavity diode lasers, locked to the $\mathrm{F}=3->\mathrm{F}$ ' $=4$ and $\mathrm{F}=2->\mathrm{F}^{\prime}=3$ transitions for the cooling and the repumper beams. The beams from each of the two lasers were split into four parts, one of which was sent to a Doppler-free saturation absorption setup in a vapour cell that was used to determine the instantaneous frequency of the laser when it was ramped or was used for locking the frequency. The remaining three beams from each laser were incident into the chamber from the the $+\mathrm{X},+\mathrm{Y}$ and $+\mathrm{Z}$ directions, and were retroreflected, to form three pairs of counterpropagating, mutually orthogonal beams, that intersected at the centre of the chamber. The experimental chamber (Fig.1), made of stainless steel, was evacuated to $2 \times 10^{-9}$ Torr using a $401 / \mathrm{s}$ ion pump. The eight faces, of the octagonal chamber and the two end caps had one viewport each. Rb atoms were obtained from a getter source. While in laser cooling experiments, the repumper beam is usually sent in one direction only, in our case the repumper beams were sent in all three directions and retroreflected. This has given rise to unexpected features, as will be discussed shortly. The diameter of the beams was about $1 \mathrm{~cm}$, and thus the molasses region was a cube of side $1 \mathrm{~cm}$. Each cooling beam was of intensity $1 \mathrm{~mW} / \mathrm{cm}^{2}$, and the repumper $0.1 \mathrm{~mW} / \mathrm{cm}^{2}$. A femtowatt detector was placed at one of the viewports and a lens system imaged the fluorescent light from the centre of the chamber onto the detector. We also studied the absorption of a weak probe, in the Doppler-free pump-probe configuration shown in Fig.1.

Fluorescence from the atoms was studied when they were driven by two fields, the cooling and the repumper laser fields. The cooling laser was locked at various detunings $\delta_{c}$ in the range $+120 \mathrm{MHz}$ (blue) to $-200 \mathrm{MHz}$ (red) with respect to the cooling transition. The detunings thus spanned a region much greater than the full hyperfine manifold $\mathrm{F}=3->\mathrm{F}$ '. At each

$\left({ }^{1}\right)$ Hyperfine levels $\mathrm{F}$ of the state $5 S_{1 / 2}$ will be denoted unprimed and those of $5 P_{3 / 2}$ as $\mathrm{F}^{\prime}$ or primed. 


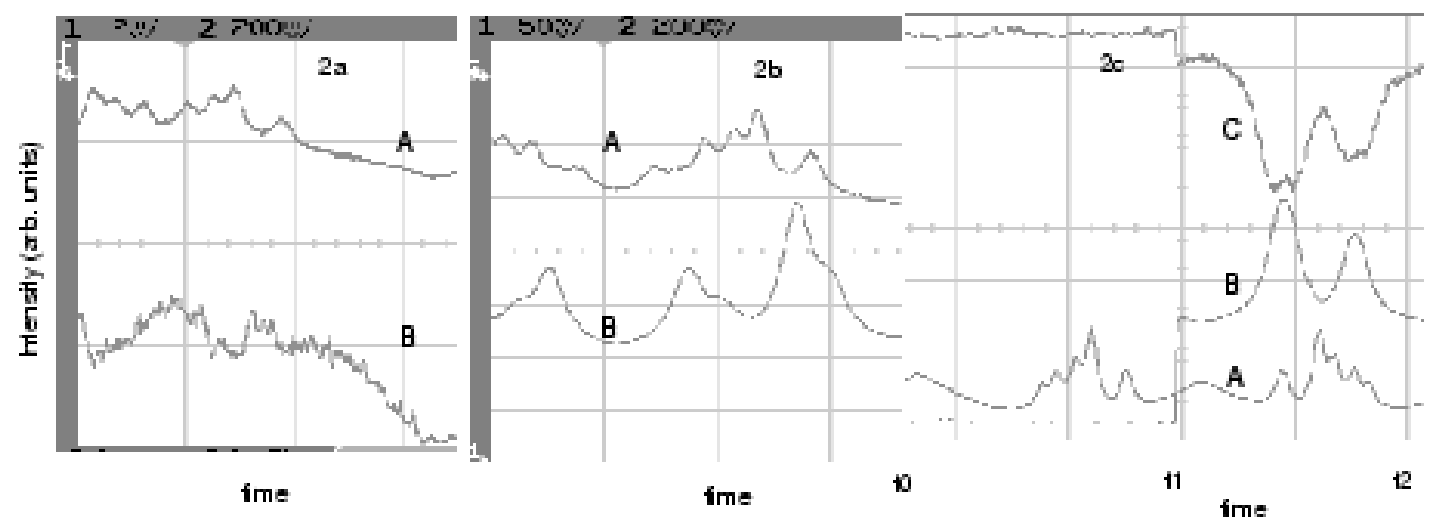

Fig. 2 - (2a) : B - Fluorescence from the molasses region when only the repumper is incident. (2b) : B - Fluorescence from the molasses region when both repumper and cooling beams are present. (2c) : B - Fluorescence from molasses region. C - Absorption of a probe (cooling laser) transiting the molasses. Trace A in all three cases is the saturation absorption signal of the frequency scanned repumper and is a measure of the instantaneous repumper frequency.

detuning the repumper laser was scanned very slowly $(0.05 \mathrm{~Hz})$ across its hyperfine manifold, i.e., $F=2->F^{\prime}$.

We remark here that cooling of atoms occurs when the cooling laser is detuned to the red within a few linewidths of the cooling transition. In our experiment, however, the detunings are often quite large, and in many cases to the blue of the cooling transition. Even though the "cooling" beam does not cool, nor does the "molasses" provide a retarding force, we retain terms like "cooling" and "molasses".

When only the repumper beam is allowed into the chamber the fluorescence is weak $(2 \mathrm{mV})$ (Fig.2a), as opposed to a stronger fluorescence $(50 \mathrm{mV})$ when the cooling beam is also incident on the atoms (Fig. 2b). All the results presented here, have fluorescence in the $50 \mathrm{mV}$ range, indicating that it is predominantly from the cooling transition $\left(4^{\prime}->3\right)$. We note that while $F=2->F^{\prime}=1,2,3$ are allowed transitions, only $F^{\prime}=2,3$ serve to repump atoms back into the cooling cycle. As we see enhanced fluorescence only when the repumper laser is close to either of these transitions (Fig.2b), it confirms that it is predominantly the cooling transition that contributes to the fluorescence. Fig. 2c illustrates an interesting feature. Trace A shows the saturated absorption spectrum of the repumper laser that is periodically scanned across $F=2->F^{\prime}$ manifold. Trace B is the fluorescence signal from the molasses and trace $\mathrm{C}$ the absorption of a weak probe derived from the cooling laser that is locked to the $F=3->F^{\prime}=4$ transition. For the first half of the trace, the repumper laser to the molasses is blocked. During this period, the fluorescence and absorption signals are unchanged in time, which is as they should be, as the cooling laser is locked. In the later half, the cooling laser continued to be locked, but the repumper, with its frequency being periodically scanned, was allowed into the molasses region. The presence of the repumper dramatically alters the absorption of the cooling laser, as is seen from trace C. Further, as trace B is roughly similar (though inverted) to trace $\mathrm{C}$, it is reasonable to conclude that the light absorbed from the cooling beam by the atom is spontaneously emitted in random directions, and forms the fluorescence signal.

The dependence of the fluorescence signal on the detunings of the two driving fields is 
shown in Fig. 3a $\left(\delta_{c}<0\right)$ and $3 \mathrm{~b}\left(\delta_{c}>0\right)$. In both figures, trace A shows the saturation absorption spectrum of the repumper as it is scanned across $F=2->$ F', providing a measure of the instantaneous repumper frequency. The remaining traces display the instantaneous fluorescence intensity collected from the molasses region, for various detunings of the cooling laser from the $\mathrm{F}=3->\mathrm{F}^{\prime}=4$ cooling transition. Trace $\mathrm{B}$ in both figures shows the fluorescence from the molasses with the cooling laser on resonance. The fluorescence shows two maxima, ( labelled ' $a$ ' and ' $b$ '), corresponding to the two repumping transitions. For small detunings of the cooling laser (Traces $3 \mathrm{a}(\mathrm{C})$ and $3 \mathrm{~b}(\mathrm{C})$ ), narrow dips appear in the fluorescence signal, splitting the peaks $a$ and $b$. These are centred at the points where the repumper laser is exactly resonant with the repumping transitions. Each of the peaks $a$ and $b$ split into a pair of peaks, labelled $a_{+}, a_{-}$and $b_{+}, b_{-}$, symmterically displaced from the original peaks, a and b. As the cooling laser is progressively detuned, each pair $a_{+}, a_{-}$and $b_{+}, b_{-}$separate further (Traces $\mathrm{D}-\mathrm{F}$ in $3 \mathrm{a}$ and $3 \mathrm{~b}$ ) resulting in four distinct peaks in the fluorescence signal. The width of each peak remains fairly constant at $30 \mathrm{MHz}$. In several cases, there appear some very weak peaks, indicated by arrows. These peaks are well resolved only for large detunings of the cooling laser. In Fig. 3c, the frequency shift of each of the four peaks with respect to the unsplit repumper transition $\left(F=2->F^{\prime}=3\right)$ is plotted as a function of the detuning of the cooling laser $\delta_{c}$. The peak positions fall on straight lines with slopes \pm 1 ; the two lines corresponding to the + and - peaks of each transition intersect when the detuning of the cooling laser is zero. The intercepts are separated by $\approx 63 \mathrm{MHz}$, which corresponds to the hyperfine interval between the upper levels $F^{\prime}=2$ and 3 of the repumping transition.

Discussion. - Typical experiments on two and three level atoms using multiple fields study the absorption of a weak probe that has counter-propagating to it a strong beam of the same frequency, that enables the selection of atoms with zero velocity along the direction of the two beams. It is in this configuration that one studies narrow spectral features, that could otherwise not have been resolved due to the Doppler shift. By the same token, fluorescence measurements are not expected to display narrow spectral features as they are emissions from a collection of randomly moving atoms. It therefore comes as a surprise that our experiment shows dips in the fluorescence as narrow as $6 \mathrm{MHz}$, separating peaks with widths of $30 \mathrm{MHz}$.

It may be noted that while we are not performing any frequency analysis of the emitted fluorescent light, we are studying the fluorescence response of the atoms to the repumper laser that is being frequency scanned. The various spectral features occur at the same relative positions to the repumper peaks for all slow rates of scan.

The narrow spectral features suggests a quantum phenomenon. In this context, one could look for Autler Townes splitting, where, due to the presence of a strong pump laser, the upper $\left({ }^{2}\right)$ levels get split. In our case, the pump laser is the cooling laser, which splits the upper repumper levels $\left(F^{\prime}=2,3\right)$. This split would be directly discernible if one were looking for absorption to, or emission from these levels. In our experiment, we are recording predominantly, the emission due to the cooling transition $\left(F^{\prime}=4 \rightarrow F=3\right)$. It is, however, possible for the Autler Townes splitting of the upper repumper levels $\left(\mathrm{F}^{\prime}=2\right.$ and 3$)$ to be indirectly reflected in the fluorescence signal from $F^{\prime}=4$ level.

In the absence of the cooling laser, when the repumper is scanned, atoms with zero velocity will make an upward transition from $F=2->3^{\prime}$ when the frequency of the laser $\omega_{23^{\prime}}$ is the transition frequency. In the presence of the strong cooling laser, the $F^{\prime}=3$ level will split, giving rise to the Autler-Townes doublet with displacements $\delta_{ \pm}=\delta_{p} / 2 \pm\left(\delta_{p}^{2}+\Omega^{2}\right)^{1 / 2} / 2[5]$. $\delta_{p}$ refers to the detuning of the pump (cooling) laser from the $\mathrm{F}=3->\mathrm{F}^{\prime}=3^{\prime}$ transition, (i.e., $\delta_{c}+2 \pi * 121 \mathrm{MHz}$ ), and $\Omega$ its Rabi frequency. Thus, when the repumper laser frequency is

$\left({ }^{2}\right)$ lower levels do not split as $\mathrm{J}=1 / 2$ 

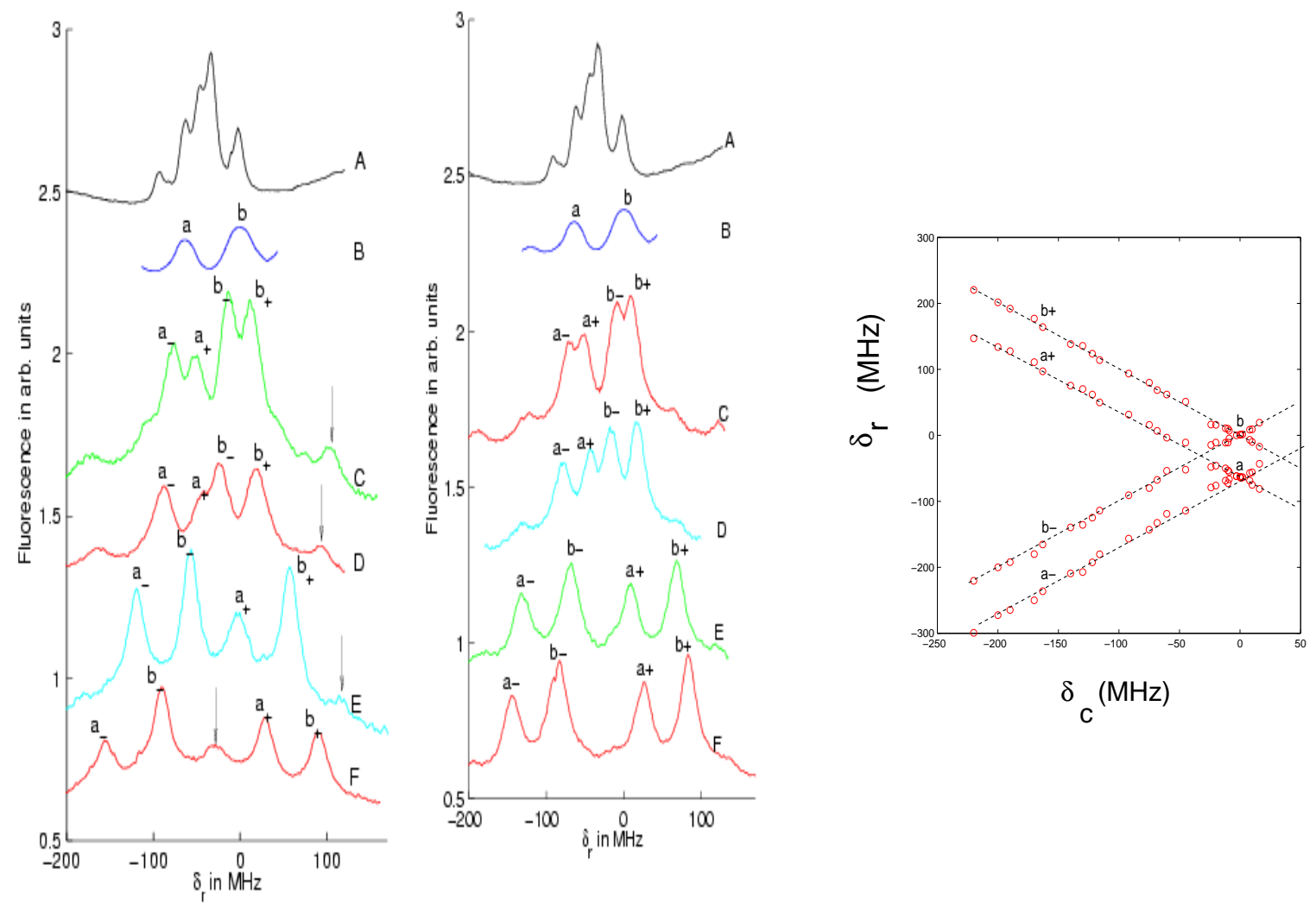

Fig. 3 - Fluorescence signal from the molasses for (3a) red detuning and (3b) blue detuning of the cooling laser as a function of the instantaneous repumper frequency. The uppermost trace gives the saturation absorption spectrum of the repumper laser. Traces B-F correspond to $\delta_{c}=0,-12,-22,-60$ and $-92 \mathrm{MHz}$ in (3a) and to $0,10,20,70$ and $90 \mathrm{MHz}$ in (3b). 3c : The positions of the fluorescence peaks as a function of the detuning of the cooling laser. The dotted lines have slopes \pm 1 and are drawn as a guide to the eye. $\delta_{r}=0$ corresponds to the $2->3^{\prime}$ transition.

scanned about $\omega_{23^{\prime}}$, atoms will be transferred out of the $\mathrm{F}=2$ level twice, when the laser is at $\omega_{23^{\prime}}+\delta_{-}$and at $\omega_{23^{\prime}}+\delta_{+}$. At both these instants, nearly all atoms will be transferred to the level $\mathrm{F}=3$ via the upper repumper level. These atoms can then participate in the "cooling" transition, resulting in an enhanced fluorescence thus giving an indirect evidence of the Autler-Townes split $\left(^{3}\right)$. In our experiment, we do indeed see maximal repumping efficiency for two values of the repumper frequency about each repumping transition. However, the peak positions are symmetrically displaced about the unsplit position which is unlike the Autler-Townes doublet, where for example, for small $\Omega$, the shifts are 0 and $\delta_{p}$. Further, the fluorescence is narrow, and not Doppler broadened as expected.

The Double Resonance Model. - Maximum fluorescence is obtained when atoms are repumped from $\mathrm{F}=2$ level to $\mathrm{F}=3$ level, and then raised to $\mathrm{F}^{\prime}=4$. This requires the enhanced

$\left({ }^{3}\right)$ The upper cooling level $(\mathrm{F}=4)$ too, should experience a similar ac Stark splitting. This, however, will not be seen in our measurements as we are not scanning the cooling laser, nor are we performing a frequency discrimination of the emitted fluorescence signal. 

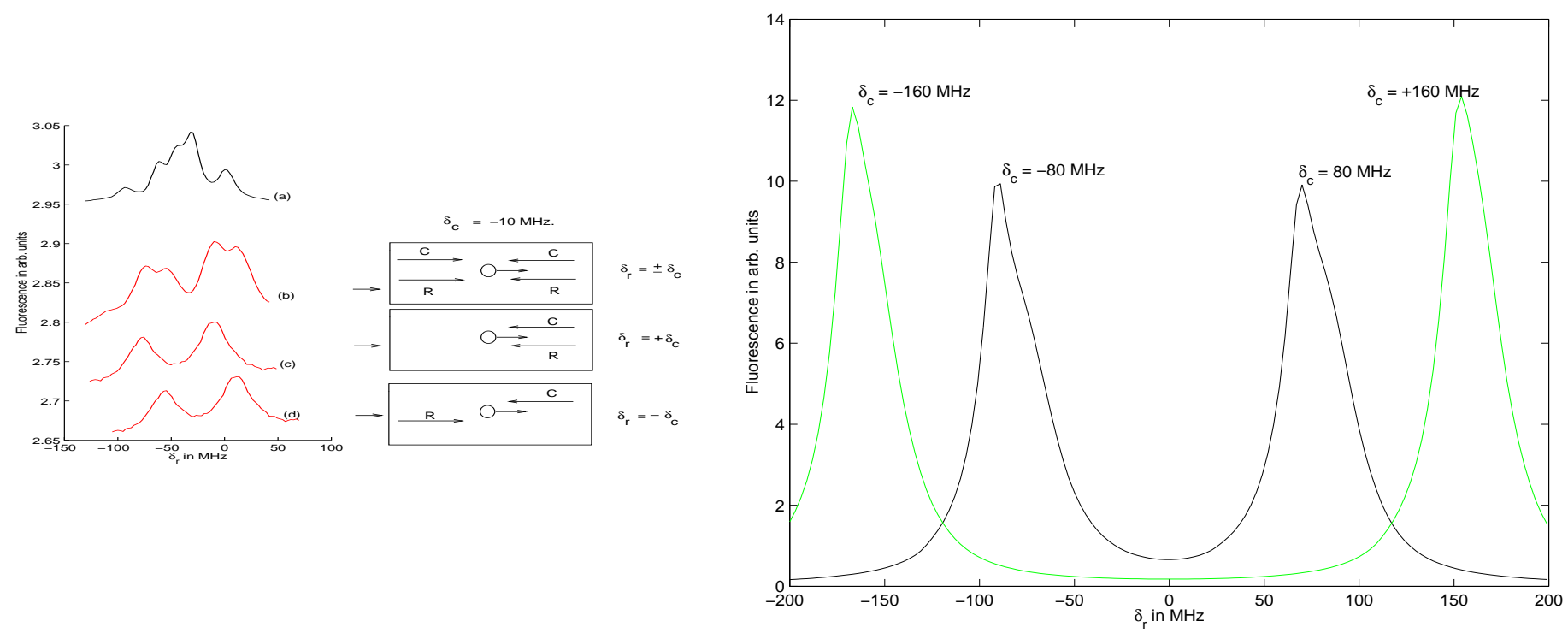

Fig. 4 - (a): Trace a: Saturation absorption signal of the repumper. Trace b,c and d are the fluorescence signals obtained for the beam configurations shown alongside. (b): Fluorescence estimated using equation (4) for $\delta_{c}=-100 \mathrm{MHz}$ (green) and $\delta_{c}=80 \mathrm{MHz}$ (black).

transition rates from $2->3^{\prime}$ and $3->4^{\prime}$ or, $2->2^{\prime}$ and $3->4^{\prime}$, or equivalently, increased absorption rates of a repumper photon and a cooling photon. The requirement that two photons be absorbed, in turn, requires that the detuning of the two lasers be compensated for by the Doppler shift. This provides a means of velocity selection where atoms of a particular velocity can be selected depending on the detuning of the pump laser. This is in contrast to the conventional Doppler-free saturation absorption, where only zero-velocity atoms are selected out.

For an atom with velocity $\vec{v}$, and cooling beam of detuning $\delta_{c}$, absorption is maximised when $\vec{k} \cdot \vec{v}=-\delta_{c}$. The repumper, which serves to bring atoms back to the cooling cycle, will be most effective for this velocity class of atoms only when its detuning $\delta_{r}= \pm \vec{k} . \vec{v}$. The '+' (-) sign is for the repumper beam counter- (co-) propagating to the cooling beam. Both possibilities exist in our experiment. If $\delta_{r} \neq \pm \vec{k} \cdot \vec{v}$ repumping will not occur and the fluorescence from the cooling transition will be diminished. This is precisely what is seen in the experiment. The slopes \pm 1 in Fig.3c indicate that the maximum fluorescence occurs only when $\delta_{c}= \pm \delta_{r}$, that is, only when the resonance condition is simultaneously satisfied for both the cooling and repumper beams. Since we have two repumper transitions we see two pairs of lines. For $\delta_{c}=0$ the two pairs of lines converge at $\delta_{r} \approx 0$ and $\delta_{r} \approx-63 \mathrm{MHz}$, this being the hyperfine interval between $\mathrm{F}^{\prime}=3$ and $\mathrm{F}^{\prime}=2$ (Fig. 3c). On the lines of [6], this suggests a means of determining hyperfine intervals to good accuracy.

To verify the validity of this model, experiments where performed for various configurations of co- and counter- propagation of the laser beams. The results are shown in Fig. 4a. When the cooling and repumper beams are both in the same direction $(\vec{k})$, an atom with velocity $\vec{v}$, sees them as $\omega_{c}+\delta_{c}-\vec{k} \cdot \vec{v}$ and $\omega_{r}+\delta_{r}-\vec{k} \cdot \vec{v}$. For the two transitions to be simultaneously on resonance, we require $\delta_{c}=\delta_{r}=\vec{k} \cdot \vec{v}$. The experiment did indeed yield a peak at $\delta_{r}=\delta_{c}$ 
only. For the case, where the repumper is counterpropagating to the cooling beam, an atom of velocity $\mathrm{v}$ will see the lasers at $\omega_{r}+\delta_{r}+\vec{k} \cdot \vec{v}$ and $\omega_{c}+\delta_{c}-\vec{k} \cdot \vec{v}$, giving the condition $\delta_{c}=-\delta_{r}$ for simultaneous resonance of the two transitions. Once again, the experiment bore this out. When the cooling and repumper beams are incident in both directions, peaks appear at both $\pm \delta_{c}$, confirming that simultaneous Doppler resonance is the origin of the symmetric pairs of fluorescence peaks. This, surprisingly, gives rise to a fluorescence spectrum of line width as narrow as $30 \mathrm{MHz}$ from a collection of atoms of Doppler width $500 \mathrm{MHz}$.

Calculation of fluorescence peaks. - We now give an analytical derivation of the fluorescence spectra to show that narrow fluorescent peaks will indeed arise upon double resonance. For simplicity, we consider beams along the $\pm \mathrm{z}$ direction only. When the atoms enter the molasses region, one half of the atoms of any velocity $v$ are in the $F=2$ level and the other half in the $F=3$ level. Within the molasses, there is a redistribution of atoms between the two ground states due to the laser fields. The rate at which the cooling beam transfers atoms from $F=3$ to $F=2$ through the upper level $F^{\prime}=3$ is given by

$$
\eta_{32}=\Gamma_{3^{\prime} 3} \frac{\frac{I_{c}}{I_{s 33^{\prime}}}}{\sqrt{1+2\left(I_{c} / I_{s 33^{\prime}}\right)+4\left(\Delta_{3^{\prime} 3} / \Gamma_{3^{\prime} 3}\right)^{2}}} \frac{\Gamma_{3^{\prime} 2}}{\left(\Gamma_{3^{\prime} 3}+\Gamma_{3^{\prime} 2}\right)}
$$

Here, $I_{c}=$ Intensity of the cooling beam, $\Gamma_{i j}=$ linewidth of the transitions between states $\mathrm{i}$ and $\mathrm{j}, I_{s i j}=$ saturation intensity of transition from i to $\mathrm{j}, \Delta_{3^{\prime} 3}=\Delta_{c}+2 \pi 121 \mathrm{MHz}, \Delta_{c}=$ $\delta_{c}-\vec{k} \cdot \vec{v}$. Similarly, the rate at which the repumper laser transfers atoms from $\mathrm{F}=2$ to $\mathrm{F}=3$ through $\mathrm{F}^{\prime}=3$ is

$$
\eta_{23}=\Gamma_{3^{\prime} 2} \frac{\frac{I_{r}}{I_{s 23^{\prime}}}}{\sqrt{1+2\left(I_{r} / I_{s 23^{\prime}}\right)+4\left(\Delta_{r} / \Gamma_{3^{\prime} 2}\right)^{2}}} \frac{\Gamma_{3^{\prime} 3}}{\left(\Gamma_{3^{\prime} 3}+\Gamma_{3^{\prime} 2}\right)}
$$

The symbols have meanings as given earlier, $\Delta_{r}=\delta_{r}-\vec{k} \cdot \vec{v}$.

Let us assume that there are $\mathrm{N}$ atoms with velocity $v$. Of these, at a time $\mathrm{t}, N_{3}$ are taken to be in level $\mathrm{F}=3$ and $N-N_{3}$ in level $\mathrm{F}=2$, neglecting any small number that may be in the upper levels $\mathrm{F}^{\prime}=3^{\prime}$ and 4 '. $N_{3}$ should satisfy the rate equation -

$$
\frac{d N_{3}}{d t}=-\left(\eta_{32}+\eta_{23}\right) N_{3}+\eta_{23} N
$$

The solution to this is

$$
N_{3}(t)=\left[N_{30}-\frac{\eta_{23}}{\eta_{23}+\eta_{32}} N\right] e^{-\left(\eta_{23}+\eta_{32}\right) t}+\frac{\eta_{23}}{\eta_{23}+\eta_{32}} N
$$

We see that the first term decays at a rate $\tau=\frac{1}{\eta_{32}+\eta_{23}}$ and the steady state value of the population in level $\mathrm{F}=3$ is given by the second term. At double resonance $\left({ }^{4}\right), \eta_{23}>>\eta_{32}$, $N_{3}$ rises rapidly from $N_{30}$ to nearly $\mathrm{N}$ with a rise-time of a few tens of microseconds, and the fluorescence is maximised. Away from double resonance, the fluorescence is much smaller, as has been estimated using equation (4) and as is shown in Fig. 4b. The fluorescence is maximum when $\left|\delta_{r}\right|=\delta_{c}$ with a halfwidth of about $25 \mathrm{MHz}$ that is governed by the velocity range of the atoms which contribute to the fluorescence effectively. This simple rate analysis gives a plausible explanation of a narrow fluorescent peaks despite the presence of a thermal velocity distribution. A rigourous density matrix calculation is given in a companion paper.

$\left({ }^{4}\right)$ If the cooling laser is seen at resonance with the cooling transition, it is then $121 \mathrm{MHz}$ away from the $3->3^{\prime}$ transition 
Conclusions. - Fluorescence from an optical molasses in ${ }^{85} \mathrm{Rb}$ in the presence of a pump (cooling) and probe (repumper) laser shows splitting of peaks, with the separation increasing linearly with the detuning of the pump. A simple model explains both the peak positions and their narrow linewidth as arising from velocity selection due to the requirement of simultaneous Doppler resonance. It also provides a means of selecting out atoms of a narrow velocity range from a hot gas.

Acknowledgements. - It is a pleasure to thank M.S. Meena for building the electronics modules, the workshop for fabricating numerous components and Prof. N. Kumar for his constant encouragement. We also thank Prof. A. Kastberg, Umea University, Sweden, for his unstinting help during the construction of the experimental setup. We are grateful to Dr. C.S. Unnikrishnan, TIFR, India, for the loan of the femto-Watt detector.

\section{REFERENCES}

[1] Andal Narayanan et. al to be published

[2] Harris, S.E., Phys. Today, 50, (1997) 36 and references therein.

[3] Zibrov, A.S. et.al, PRL $\mathbf{7 5}$ (1995),1499.

[4] Autler S.H and Townes C. H., Phys. Rev. 100 (1955),703.

[5] Vemuri G et.al, Phys. Rev. A 53 (1996), 2842. Unanyan R.G et. al, Phys. Rev. A, 62 (2000), 043407. Arimondo E and Orriols G., Nuovo Cimento letters 17 (1976) 333.

[6] Zhu Y and Wasserlanf T.N., Phys. Rev. A 54 (1996) 3653. Rapol U.D and Natarajan V., Euro. Phys. Lett. 60 (2002), 195.

[7] de Echaniz, S.R., et.al Phys. Rev. A 64 (2001),013812 